\title{
Multiple abdominal nodules in a patient with ulcerative proctitis: a case of peritoneal splenosis
}

\author{
Margherita Marocchi, ${ }^{1,2}$ Elisabetta Ascari, ${ }^{1}$ Maria Chiara Boni, ${ }^{1}$ Barbara Marani, ${ }^{1}$ Concetto Cartelli, ${ }^{3}$ \\ Ruggero Bollino, ${ }^{3}$ Francesco Rossi,${ }^{4}$ Giovanni Fornaciari ${ }^{1}$
}

${ }^{1}$ SC Medicina III e Gastroenterologia, AO Arcispedale Santa Maria Nuova, Reggio Emilia; ${ }^{2}$ Scuola di Specializzazione in Gastroenterologia ed Endoscopia Digestiva, Università di Modena e Reggio Emilia; ${ }^{3}$ SC Chirurgia II, AO Arcispedale Santa Maria Nuova, Reggio Emilia; ${ }^{4} \mathrm{SC}$ Radiologia, AO Arcispedale Santa Maria Nuova, Istituto di Ricerca e Cura a Carattere Scientifico, Reggio Emilia, Italy

\begin{abstract}
A 40-year old gardener was referred for ulcerative proctitis treated with topical mesalamine with rapid improvement of symptoms. Eighteen years before he had had a splenectomy for traumatic splenic rupture. At the end of 2010, he was admitted to another hospital because of abdominal pain. Computerized tomography (CT) scan and magnetic resonance imaging revealed multiple abdominal nodules but a definite diagnosis was not made. While being examined for the proctitis, the patient was admitted to our unit due to worsening of the abdominal pain. After another CT scan, a laparoscopy was performed: several reddish-blue nodules on the peritoneal wall were observed and biopsies were performed. Histological examination was consistent with splenosis. After the procedure, we observed an improvement in the abdominal pain. Splenosis is an acquired condition defined as autotransplantation of viable splenic tissue throughout different sites of the body. It occurs after splenic rupture via trauma or surgery. Splenosis is a benign condition that is usually found incidentally unless symptomatic. Since on radiographic examination it can mimic a neoplasia, extensive workup is usually needed. The diagnostic method of choice is nuclear scintigraphy. Splenosis usually occurs in the abdominal and pelvic cavities but patients have been described with splenosis in other intrathoracic, hepatic and subcutaneous sites. Splenosis is usually asymptomatic and treatment is not necessary. Most patients who have an exploratory laparotomy or laparoscopy for abdominal pain, such as in our patient, experience no more pain after the procedure, regardless of whether the splenic nodules have been completely removed or not. The reason for this spontaneous improvement is not known.
\end{abstract}

Correspondence: Giovanni Fornaciari, Medicina III e Gastroenterologia, Arcispedale Santa Maria Nuova, viale Risorgimento 80, 42122 Reggio Emilia, Italy.

Tel. +39.052.2296687 - +39.052.296605.

Fax: +39.052 .2295927 .

E-mail: fornaciari.giovanni@asmn.re.it

Key words: splenosis, ulcerative proctitis, laparoscopy, splenectomy.

Conflict of interests: the authors declare no potential conflict of interests. The patient gave his informed consent to the laparoscopy and to the publication of this case report.

Conference presentation: Presented as an oral communication at the $13^{\text {th }}$ Regional Congress of the Federation of Associations of Hospital Doctors on Internal Medicine (FADOI) of EmiliaRomagna, in Bologna, Italy, January 2012.

This work is licensed under a Creative Commons Attribution NonCommercial 3.0 License (CC BY-NC 3.0).

(C) Copyright M. Marocchi et al., 2013

Licensee PAGEPress, Italy

Italian Journal of Medicine 2013; 7:48-51

doi:10.4081/itjm.2013.48

\section{Case Report}

A 40-year old gardener was examined for symptoms of intermittent rectal bleeding and for ulcerative proctitis on colonoscopy. This was confirmed by histological tests since there were no macro- or microscopic changes found in other parts of the colon. Topical treatment with mesalamine gave good results with quick remission of symptoms. A study of the patient's case history showed a regular lifestyle with no alcohol or drug abuse. Eighteen years earlier, the patient had undergone splenectomy for traumatic splenic rupture. He had enjoyed good health until 2008 when he was admitted to another hospital for Pneumoccus pericarditis. However, he complained of abdominal pain and this led, towards the end of 2010, to the patient being admitted to another hospital.

On admission, abdominal computerized tomography (CT) scan showed multiple abdominal nodules attributed to lymph nodes. Subsequent magnetic resonance imaging (MRI) excluded the possibility that the mass was of lymph node origin and a neoplasia was considered. Given that symptoms had since mostly been resolved, the patient was discharged with a diagnosis of alitiasic cholecystopathy. Shortly after diagnosis of ulcerative proctitis, the patient experi- 
enced renewed abdominal pain and was admitted to hospital for tests. Biohumoral values were all within the normal range. Viral and oncological markers were negative. A further abdominal CT scan (Figure 1A and B) confirmed the presence of multiple abdominal nodules the nature of which was difficult to ascertain. Also on the basis of the preceding MRI, we could only exclude lymph node origin. Given the hypothesis of abdominal tuberculosis, we performed a Quantiferon test and thoracic CT; both were negative.

Given that symptoms persisted and tended to worsen, an explorative laparoscopy was performed. This showed multiple brownish nodular lesions on the peritoneal wall and a biopsy was carried out. Histological tests were compatible with splenosis. The postoperative course was unproblematic. There was a gradual improvement in symptoms and these were completely resolved after two months. The patient is still in follow up for ulcerative proctitis, currently quiescent with no therapy.

\section{Discussion}

Splenosis is an acquired condition defined as autotransplantation of viable splenic tissue throughout different sites of the body. ${ }^{1}$ It occurs after traumatic or iatrogenic splenic rupture, and should be differentiated from accessory spleens that are congenital and occur on the left of the dorsal mesogastrium during embryogenesis. These are found in the splenopancreatic or gastrosplenic sites. ${ }^{2}$ There are clear differences between the two conditions and these differences are not only etiological. Usually no more than six accessory spleens are
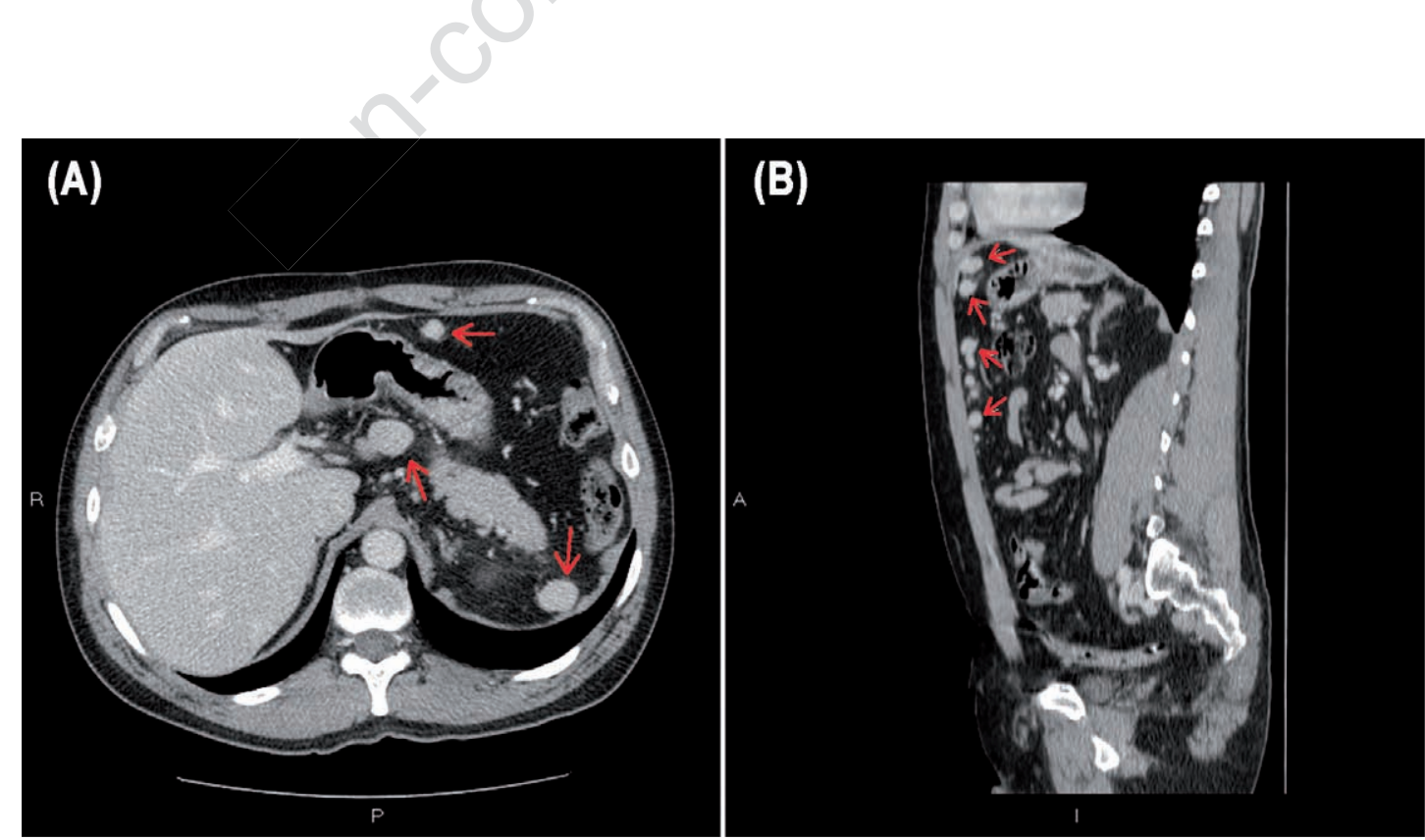

Figure 1. A and B) Abdominal computerized tomography scan showing isodense nodular foci (arrows) in the hepatic parenchymal from the portal venous phase. observed while even over a 100 splenotic nodules can be found in a single individual. ${ }^{3}$ Furthermore, accessory spleens have normal histology while splenotic nodules have irregular architecture with no hilus and with an incomplete capsule (Figures 2 and 3 ).

Vascularization also differs; accessory spleens are fed by branches of the splenic artery while splenotic nodules receive blood from small vessels in the tissue that has been penetrated. ${ }^{1}$ Splenotic nodules are nearly always found in the peritoneal wall, the omentum or the mesentery, but cases have been reported of splenosis in other sites, also extra-abdominal. The mechanism of splenosis is related to the diffusion in the peritoneal wall of splenic pulp following traumatic rupture or surgical removal of the spleen. This initiates a process of dissemination. However, hematogenous diffusion has been hypothesized in cases of extraabdominal splenosis. ${ }^{1}$ In fact it is likely that the number of splenotic nodules found is related to how much and how severely the spleen has been damaged, and also to the quantity of splenic tissues that have spread to the peritoneal wall. ${ }^{4}$ The hypothesis of hematogenous diffusion also takes into consideration the reports of some cases that, due to the presence of chronic viral hepatitis, seem to suggest a hepatocarcinoma. ${ }^{5,6}$

Abdominal or pelvic splenosis was once considered to be a rare entity. But it can now be found in $65 \%$ of cases of traumatic splenic rupture while thoracic sites account for approximately $18 \%{ }^{7}$ However, in cases of a thoracic site, traumatic rupture of the diaphragm has also been reported along with that of the spleen. ${ }^{7}$

The splenic tissue usually functions normally and guarantees, at least in part, the immune response no 
longer provided by the spleen. ${ }^{8}$ It has, therefore, been thought that patients with splenosis are at a lower risk of infection compared to those who have had a splenectomy. This is not, however, the case in our patient given that he had previously reported a serious infective complication. There is a very long average time interval between the trauma and the diagnosis of splenosis of around ten years (range 5 months- 32 years), ${ }^{9,10}$

In most cases, splenosis is diagnosed incidentally following echography or radiological examination carried out for other reasons. Symptoms such as abdominal pain and diarrhea are sometimes reported. Rarely, cases are reported of intestinal infarction or obstruction due to compression by the nodules, internal hemorrhage due to rupture of the nodules, or hydronephrosis secondary to compression. . $^{3,11,12}$

An understanding of this pathology is important above all because of the differential diagnosis with masses of neoplastic origin. As in our patient, multiple abdominal masses can suggest malignant neoplasms, lymphoma, lymphonodal metastasis or peritoneal car-

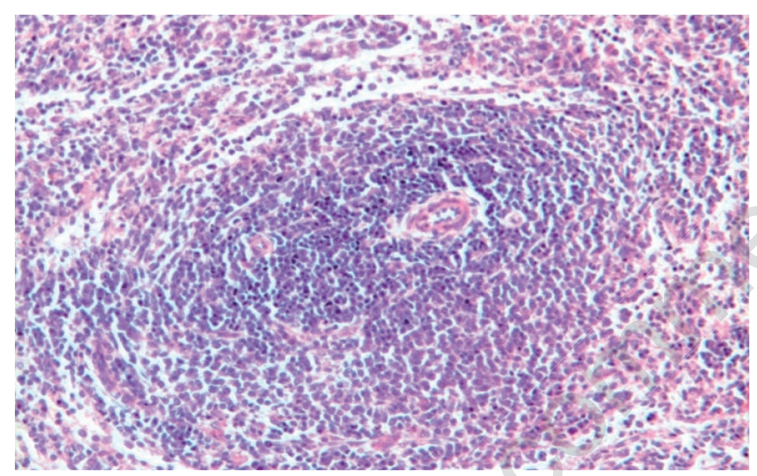

Figure 2. Histological analysis of normal spleen with fibromuscular capsule, trabecular structure, white and red pulp.

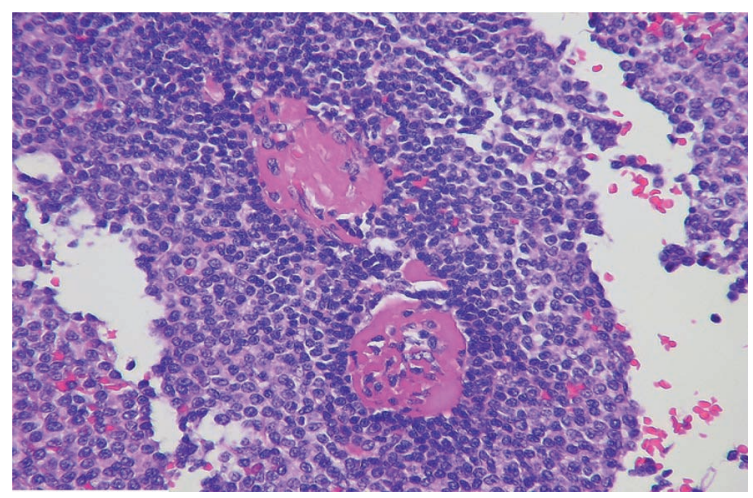

Figure 3. Histological analysis of splenotic nodule with irregular architecture, ilus absent, capsule often incomplete, normal red pulp, scarce white pulp, trabeculae absent. cinosis and, in adult females, endometriosis. ${ }^{4,13}$ Instead, splenosis, has a completely benign clinical course and it is not necessary to remove the nodules in asymptomatic patients. Furthermore, in cases in which there remains a doubt as to a possible neoplastic disease, as in our case, histological diagnosis by laparoscopy or explorative laparotomy is recommended. ${ }^{14}$

Splenotic nodules cannot usually be seen on standard radiographic examinations because of the low density of the splenic tissue. CT scans can identify the nodules and confirm their number, site and size; however, CT findings cannot be considered conclusive. Neither can MRI confirm diagnosis. Paramagnetic resonance and magnetic circular dichroism with ferumoxide has been suggested for use in the diagnosis of splenosis. ${ }^{10}$

The imaging method of choice is, however, scintigraphy with $\mathrm{Tc} 99 \mathrm{~m}$ colloid or with erythrocytes marked with Tc99m or with platelets marked with Indio-111. ${ }^{1}$ Scintigraphy with marked erythrocytes has a high level of sensitivity even in cases of limited splenosis. ${ }^{15}$ Once a diagnosis of splenosis has been confirmed with certainty, no treatment is needed as long as the patient remains asymptomatic. However, urgent treatment is needed in cases of obstruction, hemorrhage or other complications. Interestingly, as in cases like ours, an improvement in clinical condition can be observed once the patient has undergone an explorative laparotomy, even if the nodules have not been removed and only a biopsy has been carried out. ${ }^{1}$ The reason for this spontaneous improvement is not known.

\section{Conclusions}

Splenosis should be part of a differential diagnosis when abdominal masses are observed in patients who have previously undergone splenectomy or splenic surgery. Nuclear medicine tests can confirm diagnosis although, if any doubt remains, histological analysis is always recommended.

\section{References}

1. Fremont RD, Rice TW. Splenosis: a review. South Med J 2007;100:589-93.

2. Halpert B, Gyorkey F. Lesions observed in accessory spleens of 311 patients. Am J Clin Path 1959;32:165-68.

3. Al-Ahmadi M, Brundage S, Brody F, et al. Splenosis of the mesoappendix: case report and review of the literature. J R Coll Surg Edith 1998;43:200-2.

4. Brewster DC. Splenosis: report of two cases and review of literature. Am J Surg 1975;126:14-9.

5. Choi GH, Ju MK, Kim JY, et al. Hepatic splenosis preoperatively diagnosed ad hepatocellular carcinoma in a patient with chronic hepatitis B: a case report. J Korean Med Sci 2008;23:336-41.

6. Menth M, Hermann K, Haug A, et al. Intra-hepatic 
splenosis as an unexpected cause of a focal liver lesion in a patient with hepatitis $\mathrm{C}$ and liver cirrhosis: a case report. Cases Journal 2009;2:8335.

7. Huang AH, Shaffer K. Cases 93: thoracic splenosis. Radiology 2006;239:293-96.

8. Pearson HA, Johnston D, Smith KA, et al. The bornagain spleen: return of splenic function after splenectomy for trauma. N Engl J Med 1978;298:1389-92.

9. Gruen DR, Gollub MJ. Intrahepatic splenosis mimicking hepatic adenoma. Am J Roengenol 1997;168:725-6.

10. Berman AJ, Zahalsky MP, Okon SA, et al. Distinguishing splenosis from renal masses using ferumoxide-enhanced magnetic resonance imaging. Urology 2003;62: 748-8.

11. Sirinek KR, Livingston CD, Bova JG, et al. Bowel ob- struction due to infarcted splenosis. South Med J 1984;77:764-7.

12. Basile RM, Morales JM, Zupanec R. Splenosis: a cause of massive gastrointestinal hemorrhage. Arch Surg 1989;124:1087-9.

13. Fleming CR, Dickson ER, Harrison EG Jr. Splenosis: autotransplatation of splenic tissue. Am J Med 1976;61:414-9.

14. Bizekis CS, Pua B, Glassman LR. Thoracic splenosis: mimicry of a neurogenic tumor. J Thor Cardiovasc Surg 2003;125:1155-6.

15. Gunes I, Yilmazlar T, Sarikaya T, et al. Scintigraphic detection of splenosis: superiority of tomographic selective spleen scintigraphy. Radiology 1994;49:115-7. 